\title{
Research on the adaptive control method with continuous feedback parameters for controlling of chaotic motion
}

\author{
Qin $\mathrm{Wu}^{1, \mathrm{a}}$ \\ ${ }^{1}$ School of Mechanical and Electronical Engineering, Lanzhou University of Technology, \\ Lanzhou, China \\ a1261099906@qq.com
}

Keywords: feed system; the chaotic motion; adaptive control

\begin{abstract}
Although the feed system of CNC machine tool is a certain dynamic system, the chaotic motion often occurs because of the function of the nonlinear factors. The dynamic model of the feed system with nonlinear spring force and nonlinear friction force is numerically simulated by using the adaptive control method with continuous feedback parameters. It can be seen that when the system is in chaotic vibration state, the adaptive feedback control is applied, and the different target orbital period are given, that is the different time delay values $\tau$.With the adjustment of the intensity of the control signal $\sigma$, the chaotic vibration can be entered into the quasi periodic state in a short time.
\end{abstract}

\section{Introduction}

The dynamic response of the coupling effect of nonlinear factors such as stiffness and friction force of the numerical control feed system is the nonlinear dynamic equation with the forced nonlinear oscillator of the non-deterministic parameters, and with square and cubic term[1] ${ }^{*}$ The dynamic characteristics of these nonlinear dynamic equations are complex and changeable, and it is necessary to have an effect on the accuracy and surface quality of NC machining. Although the feed system of numerical control machine tool is a certain dynamic system, the chaotic motion often occurs because of the function of nonlinear factors. Chaotic motion caused the relative position relationship between the workpiece and tool disorder in NC machining process, the multi-period and broadband characteristics of chaotic motion are easy to reach the natural frequency of the system and make the system have the main resonance and sub resonance, and the paroxysmal and long-term non predictable characteristics of the chaotic motion make the motion compensation difficult[2]. In short, the chaotic motion will seriously affect the quality of numerical control processing, we must find countermeasures to control chaos.

\section{Control theory of chaotic motion}

How to transform the chaotic motion of the system into regular motion is the purpose of controlling the chaotic motion. Because of the complex motion style of the chaotic system, the traditional control method is difficult to control the chaotic system directly. E.Ott et al. [3] very well controlled the chaotic motion use the sensitivity of chaotic system to parameter variation and the dense nature of unstable periodic orbit of the chaotic attractor. The study of chaos control is gradually active, the adaptive control method, parameter periodic perturbation method, periodic excitation force method, OPF control method, delivery and transfer control method, etc. have been emerged.

The parameter adaptive control algorithm, which is proposed by Huberman[4], is used to control the chaotic system with complex oscillation state. It is controlled by the relationship between the target output and the actual output to control the parameters, make the system from chaotic motion to regular motion. Huberman uses the compound function relation of the error and the corresponding derivative, and the parameter adaptive control law and the algorithm are derived. Sinha[5] extended the adaptive algorithm of literature [4] to multiple parameters and high dimensional nonlinear systems. In the condition of the dynamic behavior changes caused by a 
sudden disturbance of system parameters suffered, the adaptive control algorithm[6] proposed by Sinha for the perturbed system restoring to the initial dynamic behavior is significantly effective, and that in any case, recovery time and control stiffness into the results of linear inverse relationship (in terms of weak stiffness), effectively control system to the equilibrium point or limit cycle state. But the above two methods are not suitable to control the movement state of the system to an unstable orbit, and the size of the control stiffness is not easy to determine, the range of the initial value of the disturbed parameters is also limited. Based on the literature [5,6], a parameter adaptive control algorithm based on reference model is proposed by Vassiliadis[7], a discrete deterministic nonlinear dynamic system model is established. The control forms of the parameters adaptive are presented, and the coupling style between the system and the model is considered. However, the control algorithm for continuous chaotic systems is not given.

\section{Adaptive control method for continuous feedback parameters}

Feeding system is a chaotic dynamical system with time continuity. The dynamic model [8]coupling with the nonlinear spring force and the nonlinear friction force is:

$$
\ddot{x}-\varepsilon \dot{x}\left(1-\dot{x}^{2}\right)+\omega_{0}^{2} x-\lambda \omega_{0}^{2} x^{3}=f_{0} \sin \omega t-u_{s} g
$$

In the equation:

$\varepsilon-$ - damping attenuation coefficient of friction nonlinear system;

$\omega_{0}$ - natural frequency of the system;

$f_{0}$ — — exciting force amplitude of unit equivalent mass;

$u_{s}$ - - static friction coefficient;

$\lambda$ - nonlinear coefficient;

$\omega$ - excitation frequency.

The model is changed into the form of state equation:

$$
\left\{\begin{array}{c}
\dot{x}=y \\
\dot{y}=\varepsilon\left(1-y^{2}\right) y-\omega_{0}^{2} x+\lambda \omega_{0}^{2} x^{3}+f_{0} \sin z-u_{s} g \\
\dot{z}=\omega
\end{array}\right.
$$

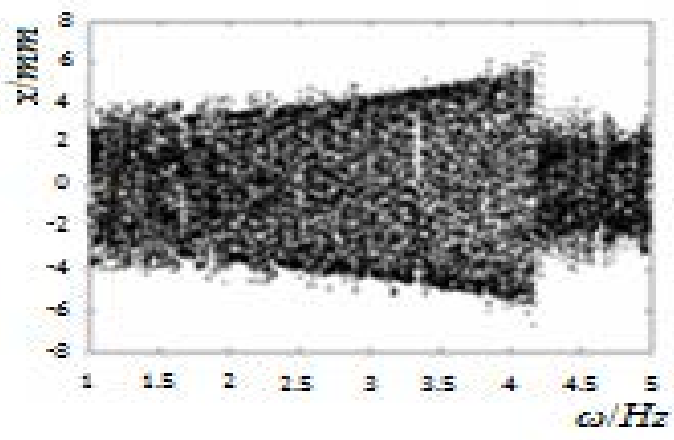

Fig.1 The bifurcation diagram of coupled svstem

When $\varepsilon=0.15, \lambda=0.6 f_{0}=50, \omega_{0}=10$, the bifurcation diagram of the coupled system is shown in Figure 1. From Figure 1 it can be recognized that the state is in a chaotic state. To suppress the chaos, make it into a periodic motion state, using continuous feedback control. We know that there are many unstable periodic orbits in the chaotic attractor, by the OGY thought, the continuous control signal $f(\mathrm{t})$ can be used to make the chaotic motion state of the system stabilize in an unstable periodic orbit in the attractor. $x$ is a measurable quantity, $y(t-\tau)$ is used to represent the unstable periodic orbit of the period Ti in the attractor, make the control signal 


$$
f(t)=\sigma[y(t-\tau)-y(t)]
$$

In the equation: $f(\mathrm{t})$ is the input control quantity, $\sigma$ is the control signal strength, $\tau$ is the lag time length, so can be obtained the equation that is the formula (2)being controlled:

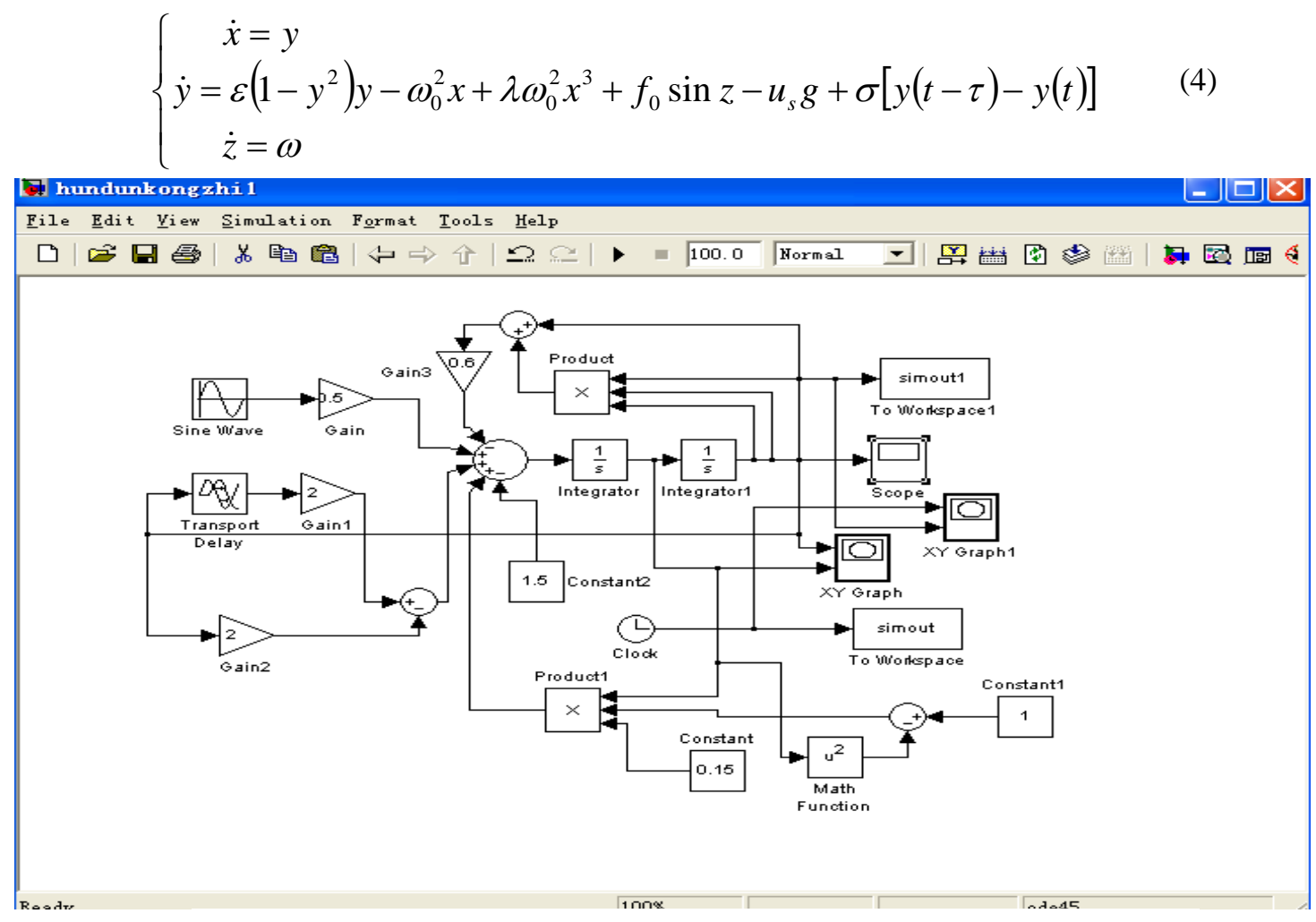

Fig.2 The principle diagram of adaptive delayed feedback control of coupled system

If $\tau$ is an unstable periodic orbit $T i$, when the system working along the track, $y(t-\tau)=y(t)$, at this time $f(t)=0$. When the $y(t)$ is just out of the track, $y(t-\tau)$ still on this track, the controller $f(\mathrm{t})$ controls the system to adjust $y(t)$ to the track. The value of $\sigma$ can be determined with the help of the calculation of Lyapunov index, those values of $\sigma$ not more than zero corresponding the maximum Lyapunov index can make the system control stabilize to the desired orbit. The magnitude of the amplitude of $f(t)$ is related to the accuracy of the reconstructed periodic orbit, the stability of the periodic orbit and the magnitude of noise disturbance.

The system can be controlled to the state of a longer period of time (the cycle is 1) by proper selection of parameters $\sigma$, and the system can be controlled to a stable periodic orbit by continuous feedback control method. This control method is more convenient and practical than the OGY method, it can control at any time of the state of the system, and the size of the control signal is not limited.

This control method includes two aspects: one is to real-time adjusting the output time delay parameter $\tau$, so that the $\tau$ step by step close to the target period is to be controlled. Two is to adaptive adjust the intensity of the control signal $\sigma$, so that the chaotic motion is gradually close to the target track, and finally search to an optimal control signal strength value $\sigma$. The adaptive control is realized by the program automatically, and the control principle is shown in Figure 2. 


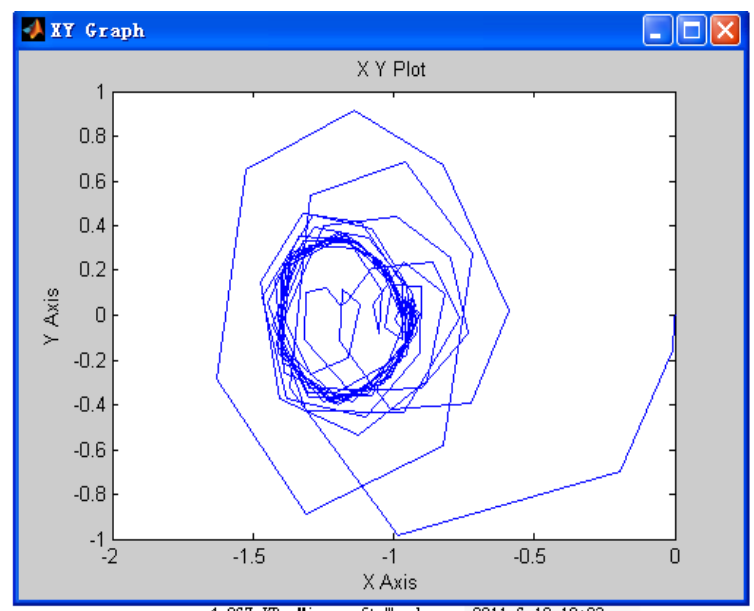

Fig.3 The phase trajectory diagram of the system without controlling

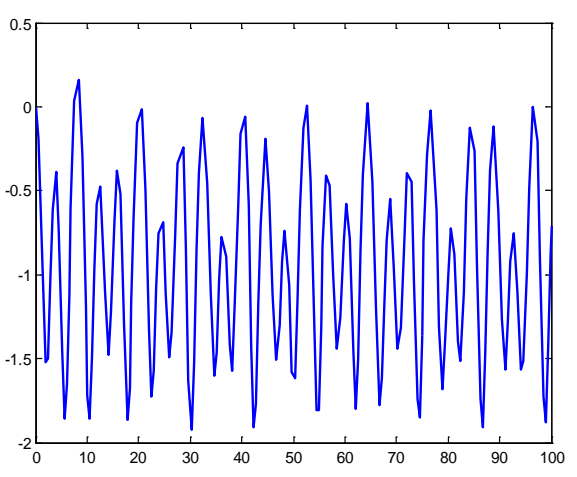

Fig. 5 The time history diagram of the system without controlling

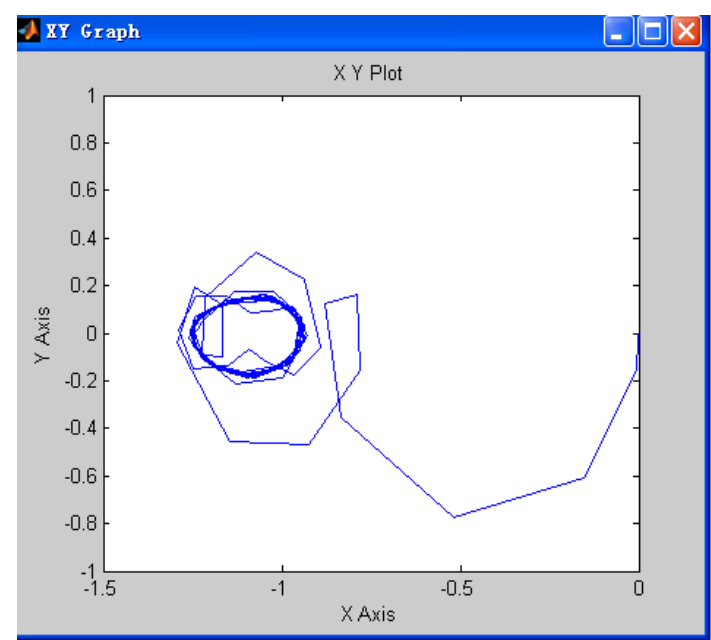

Fig.4 The phase trajectory diagram of the system after controlling of the system after controlling

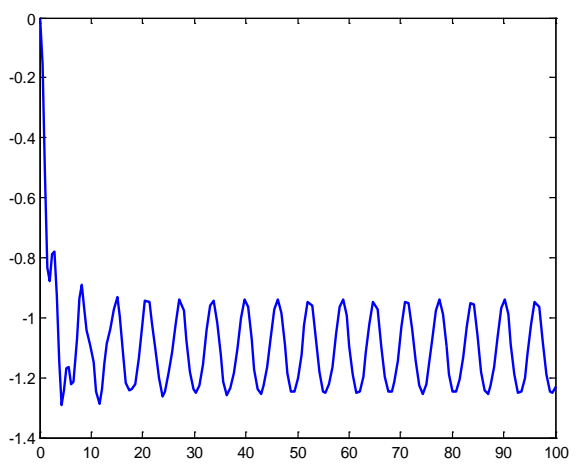

Fig.6 The time history diagram of the system after controlling

A continuous feedback parameter adaptive delay feedback method is used to simulate the dynamic equation. The simulation results of the Figure $3 \sim$ Figure 6 are obtained. From the above simulation results, we can see that when the system is in a state of chaotic vibration, applying the adaptive feedback control, give different target periodic orbits, i.e. different time delay value $\tau$, along with the regulation of the strength of control signal $\sigma$, make the vibration from the chaotic vibration to period 1, period 2 and high cycle of quasi periodic state in a relatively short period of time.

\section{Conclusion}

The effect of the chaotic motion on the processing stability is analyzed, and the method of controlling chaos is introduced in the paper as an example of adaptive control method for continuous feedback parameters. The numerical simulation results show that for the feed system that considering the coupling effect of nonlinear spring force and the nonlinear friction force, the method is effective and superior that controls the chaotic motion of the system to the low period and high periodic states.

\section{Reference}

[1] Wu Q, Rui Z Y, Yang J J.Analysis of Bifurcation and Chaos Characteristics for Ball-screw System Considering its Nonlinear Elastic Force.Journal of XI'AN Jiaotong University(Natural 
Science Edition),2012, 46(1):70-75

[2] $\mathrm{Hu} \mathrm{H}$ Y. Active Control of Chaos of Mechanical Systems.Advances in Mechanics,1996,26(4):453-463

[3] Ott E. Chaos in Dynamic Systems. New York:Cambridge University Press,1993:277-291

[4] Huberman B A, Lumer E. Dynamics of adaptive systems. IEEE Transactions on Circuits and Systems.1990,37 (4):547-550

[5] Sinha S. An efficient control algorithm for nonlinear systems. Physics Letters A, 1991 (156): 475-478

[6] Sinha S, et al. Adaptive control in nonlinear dynamics. Physica D, 1990(43):118-128

[7]Vassiliadis D. Parametric adaptive control and parameter identification of low-dimensional chaotic systems. Physica D, 1994 (71):319-341

[8] Wu Q.The Study on Dynamics Characteristics and Stability of Feeding System in Big-inertia.Lanzhou:Lanzhou University of Technology, 2012 